\title{
Counting on Community in Cyberspace
}

\author{
Marc A. Smith, Steven M. Drucker \\ Microsoft Research, \\ One Microsoft Way, \\ Redmond, WA 98052 \\ +1-425-936-6896 \\ \{masmith, sdrucker\}@microsoft.com \\ Steven Drucker, Microsoft Research \\ Robert Kraut, Carnegie Mellon University \\ Marc Smith, Microsoft Research \\ Barry Wellman, University of Toronto
}

\begin{abstract}
In this panel, a group of researchers, each of whom has either constructed tools for communicating and community building in cyberspace or studied the process, present their own work and critically evaluate each other's work. Our perspective explores the relationships between individual usage of these tools and the formation of lasting social relationships and groups. Each panelist presents a current research project that documents the empirical patterns of use and interaction with existing forms of social cyberspaces. The panelists comment on the ways in which the their findings cast new perspective on each other's findings and on the prospects for the formation of both online communities and physical communities augmented by online interaction.
\end{abstract}

\section{Keywords}

Social cyberspaces, virtual communities, social issues, metrics, World Wide Web (WWW), telecommunications

\section{COMMUNITIES IN CYBERSPACE}

Cyberspace is the site of a burgeoning collection of social relationships. Many of these relationships aggregate into collections that take on the form of communities. But very little is known about the actual patterns of use and internal behavior of individual participants or the ways their activity aggregates into larger structures and dynamics.

What constitutes a "community" in cyberspace remains an open question. Our proposal is to address this question by presenting empirical studies of existing examples of online social aggregations. We wish to accomplish two goals: First, to gather a range of base-line measures of a range of forms of social cyberspaces and to discover the aspects of community that are the glue that hold cyberspace communities together. Second, to provide guidelines for the design of new tools for community construction by providing a description of aspects of community known to be desirable.

\section{MEASURING COMMUNITIES IN CYBERSPACE}

Each of the panelists has chosen some aspect of group membership or community activity in an existing online community and gathered and studied the patterns of behavior and action that takes place with in it.

\section{Netscan: Visualizing Usenet Structure}

Panelist Marc Smith (Microsoft Research) has built Netscan (netscan.research.microsoft.com) a system for the dynamic construction of visualizations of one of the largest and most dynamic of the Internet based communities: Usenet. This system is used as a research tool for exploring the structure and dynamics of groups forming within social cyberspaces. Netscan allows users to visualize the relationships between Usenet newsgroups and between threads and posters within individual groups. Using Netscan users can track the daily changes in activity in specific groups or groups of groups. What emerges is a picture of the landscape of activity across the Usenet as a whole.

Smith's perspective on the emergence of communities in cyberspace is sociological. He draws from the extensive research on communities and collective action to model behavior within Usenet. It is well established that the interests of individuals do not always align with those of groups. When this happens a tragedy of the commons, a situation when everyone is worse off than they would have been if they had cooperated for the common good, can result. Usenet is widely viewed as having succumbed to this tragedy. Smith's research shows that, to the contrary, islands of cooperative behavior do exist in the Usenet. He proposes a range of changes to the interfaces used to access Usenet as a way to improve the quality of interaction and extend the scope of cooperative relationships. 


\section{Graphical Chats}

Panelist Steven Drucker (Microsoft Research) studies the behaviors of groups of people in graphical chat. Although graphical chat systems have been around for a number of years, there have been very few quantitative or even qualitative studies done on the use of them.

Work will be presented that analyzes the usage of V-Chat, a graphical chat system created over 2 years ago by the Microsoft Virtual Worlds Group. Graphical systems like $V$-Chat model a familiar but often unnoticed aspect of social interaction: physical distance. In all human societies the space between people conveys meaning about the nature of the relationship between participants.

This research shows, among other results, that the graphical portions of the system were used extensively even without any direct affordances (i.e. there were no explicit reasons to use the graphical portions of the system). This work addresses the question as to why people use the graphics capabilities present in V-Chat in particular and how to improve communication and community in general with innovative additions to conventional chat systems.

\section{NetVille: The Wired Suburb}

Barry Wellman's team is doing a user study of home and community use of network communication in a wired suburb north of Toronto. This suburb is unique in that Bell and Nortel have provided the homes with free high speed, wide bandwidth connectivity with (a) the Internet, (b) videophone, (c) value-added online services (such as health information, downloadable music, shopping). The first 120 residents have already moved in, with a total of 400 homes expected.

This project enables Wellman and his team to do a proper analysis of how people use existing online services, what kinds of services they want in the future, and how using these tools affects their home life. This community can be seen as a natural laboratory and window on the future, providing now the sort of wired existence that will be more prevalent five to ten years from now. The research involves two surveys of residents, 1 and 2 years after they have moved in, as well as surveys of a control group of residents moving into a similar (but unwired) suburb currently being built near-by. In addition to the surveys, a researcher on the project, Keith Hampton, has moved into the development and begun ethnographic participant-observation of life in Netville. Furthermore, logs of online use are being collected and analyzed.

\section{HomeNet: Pittsburgh's Newly Wired}

The Internet has the potential to make us socially isolated, lonely and depressed, according to the HomeNet study of home computer users performed by Robert Kraut and a research team part of Carnegie Mellon's Human-Computer Interaction Institute. The study examines the impact the Internet is having on the social involvement and psychological well being of average Americans. The findings provide a consistent picture of the downside of using the Internet extensively as a source of information or setting for friendship and or social support.

Even though people in the study heavily used electronic mail and other communication services on the Internet, the research finds that spending time on the Internet was associated with later declines in talking among family members, reductions in the number of friends and acquaintances they kept up with, and increases in depression and loneliness.

HomeNet studied 169 personal computer users in Pittsburgh, whose communications on the Internet were monitored during their first years online. A little over half the subjects are female users, a quarter of them belong to minorities. The subject pool also represents a fairly wide income range. The home computer users are families with a wide range of demographic backgrounds whose common bond was a high school age student or membership in a community development group.

Of the different demographic groups, teenagers seem the most vulnerable to potential negative effects. What's more, teenagers used the Internet for more hours than did adults. The technology that has allowed people to keep in touch with distant family members and friends, to find information quickly and to develop friendships with people around the world apparently is also replacing vital, everyday human communication. Greater use of the Internet was associated with statistically significant declines in the social involvement within a participant's families, the size of a person's social networks and reports by participants of increases in loneliness and depression, psychological states associated with reduced social involvement.

\section{ACKNOWLEDGMENTS}

Marc Smith would like to thank the Microsoft Research Virtual Worlds Group for their generous support of the Netscan project.

\section{WEB SITES}

http://netscan.research.microsoft.com

(Netscan Usenet Visualization System)

http://vchat.microsoft.com

(Microsoft V-Chat)

http://homenet.andrew.cmu.edu

(HomeNet Study)

\section{REFERENCES}

1. Axelrod, Robert, 1984. The Emergence of Cooperation, New York: Basic Books

2. William H. Whyte, 1971 City : Rediscovering the Center, New York: Anchor Books

3. Herbert Gans, 1982 The Levittowners: Ways of Life and Politics in a New Suburban Community, New York: Columbia University Press

4. Edward Twitchell Hall, 1990, The Hidden Dimension, New York: Anchor Books

5. Erving Goffman, 1976. Relations in Public, Harper Colophon, New York. 